\title{
The LORIS MyeliNeuroGene Rare Disease Database for Natural History Studies and Clinical Trial Readiness
}

\section{Aaron Spahr}

McGill University Health Centre

\section{Zaliqa Rosli}

McGill University Health Centre

\section{Mélanie Legault}

McGill University Health Centre

\section{Luan Tran}

McGill University Health Centre

\section{Simon Fournier}

McGill University Health Centre

Helia Toutounchi

McGill University Health Centre

\section{Lama Darbelli}

McGill University Health Centre

\section{Cecile Madjar}

McGill University Health Centre

\section{Cassandra Lucia}

McGill University Health Centre

Marie-Lou St-Jean

McGill University Health Centre

\section{Samir Das}

McGill University Health Centre

\section{Alan Charles Evans}

McGill University Health Centre

Geneviève Bernard ( $\square$ genevieve.bernard@mcgill.ca )

McGill University Health Centre https://orcid.org/0000-0002-9634-2966

\section{Research Article}

Keywords: leukodystrophy, rare diseases, information management systems, databases, registry, natural history, outcome measures, clinical trials, biomarkers 
Posted Date: May 6th, 2021

DOl: https://doi.org/10.21203/rs.3.rs-491571/v1

License: (9) This work is licensed under a Creative Commons Attribution 4.0 International License. Read Full License

Version of Record: A version of this preprint was published at Orphanet Journal of Rare Diseases on July 23rd, 2021. See the published version at https://doi.org/10.1186/s13023-021-01953-8. 
The LORIS MyeliNeuroGene Rare Disease Database for Natural History Studies and Clinical Trial Readiness

Aaron Spahr ${ }^{1,2,3,4,5}$, Zaliqa Rosli ${ }^{6}$, Mélanie Legault ${ }^{6}$, Luan $\mathbf{T}$ Tran $^{1,2,3,4,5}$, Simon Fournier $^{1,2,3,4,5}$, Helia Toutounchi ${ }^{1,2,3,4,5}$, Lama Darbelli ${ }^{1,2,3,4,5}$, Cécile Madjar, Cassandra Lucia $^{1,2,3,4,5}$, Marie-Lou St-Jean ${ }^{1,2,3,4,5}$, Samir Das $^{6}$, Alan C Evans $^{6}$, Geneviève Bernard $^{1,2,3,4,5,+}$

1. Department of Neurology and Neurosurgery, McGill University, Montréal, Québec Canada

2. Department of Pediatrics, McGill University, Montréal, Québec, Canada

3. Department of Human Genetics, McGill University, Montréal, Québec, Canada

4. Department of Specialized Medicine, Division of Medical Genetics, McGill University Health Centre, Montréal, Québec, Canada

5. Child Health and Human Development Program, Research Institute of the McGill University Health Center, Montréal, Québec, Canada

6. McGill Centre for Integrative Neuroscience, Montreal Neurological Institute, McGill University, Montréal, Québec, Canada

+ Corresponding Author: genevieve.bernard@mcgill.ca

\section{Abstract}

Background: Rare diseases are estimated to affect 150-350 million people worldwide. With advances in next generation sequencing, the number of known disease-causing genes has increased significantly, opening the door for therapy development. Rare disease research has therefore pivoted from gene discovery to the exploration of potential therapies. With impending clinical trials on the horizon, researchers are in urgent need of natural history studies to help them identify 
24 surrogate markers, validate outcome measures, define historical control patients, and design 25 therapeutic trials. Results: We customized a browser-accessible multi-modal (e.g. genetics, 26 imaging, behavioral, patient-determined outcomes) database to increase cohort sizes, identify 27 surrogate markers, and foster international collaborations. Ninety data entry forms were developed 28 including family, perinatal, developmental history, clinical examinations and diagnostic 29 investigations, neurological evaluations (i.e. spasticity, dystonia, ataxia, etc.), disability measures, parental stress, and quality of life. A customizable clinical letter generator was created to assist in

31 continuity of patient care. Conclusions: Small cohorts and underpowered studies are a major 32 challenge for rare disease research. This online, rare disease database will be accessible from all over the world, making it easier to share and disseminate data. We have outlined the methodology to become Title 21 Code of Federal Regulations Part 11 Compliant, which is a requirement to use electronic records as historical controls in clinical trials in the United States. Food and Drug Administration compliant databases will be life-changing for patients and families when historical control data is used for emerging clinical trials. Future work will leverage these tools to delineate the natural history of several rare diseases and we are confident that this database will be used on a larger scale to improve care for patients affected with rare diseases.

Keywords: leukodystrophy, rare diseases, information management systems, databases, registry, natural history, outcome measures, clinical trials, biomarkers

\section{Background}

According to the World Health Organization (WHO), the definition of a rare disease is one that affects every 1 in 2,000 people or less. Global prevalence of these approximately 8,000 rare genetic disorders is estimated to affect between 150 and 350 million people [1-7]. Historically, rare diseases have been notoriously difficult to diagnose due to their heterogeneous phenotypes 
48 and genotypes [8]. Since only around 5\% of all rare diseases have an FDA-approved treatment, 49 many orphan diseases utilize off-label indications of medications approved for other purposes [9].

50 However, an incredible amount of advancement has been accomplished over the last decade using 51 rapidly evolving genetic technologies, including with the most recent use of next generation 52 sequencing (NGS), to identify genes causing these diseases [6]. The description of novel rare 53 disease entities and the identification of novel disease-causing genes have opened the door for 54 studies investigating disease pathogenesis and potential therapeutic approaches [6]. Rare disease 55 research has therefore pivoted from gene discovery towards investigating potential treatments [6].

With impending clinical trials on the horizon, rare disease researchers are realizing a 57 tremendous need for natural history data $[10,11]$. The goal of a natural history study is to recruit 58 patients for longitudinal analysis of natural disease progression [12]. The data gathered is used to 59 help identify surrogate markers, determine the best outcome measures to be used in potential 60 therapeutic trials, and can serve as the control arm of a clinical trial [13-16]. Natural history studies 61 result in incredible amounts of information being collected, including clinical, behavioral, 62 sociodemographic, genetic, imaging, and patient and family reported outcomes. This diversity and quantity of data can be difficult to manage, so rare disease researchers 64 must begin to utilize information management systems, or databases, to facilitate natural history 65 studies. Rare disease research relies heavily on international collaboration and data sharing in order 66 to recruit large patient populations to obtain adequate statistical power [6, 17]. Therefore, utilizing 67 an online database can uniquely benefit rare disease research more than other disease research 68 fields where significant patient populations are more prevalent. If rare disease databases are going to be successful in future clinical trials, they must adhere 70 to local and international regulations for electronic records. Title 21 Code of Federal Regulations 
71 (CFR) Part 11 published in 1997, from the U.S. Food and Drug Administration, outlines what is

72 considered trustworthy, reliable record keeping. These regulations apply to any FDA-regulated

73 industry, such as pharmaceutical companies, medical device manufacturers, biotechnological

74 companies, and clinical research organizations. We chose to adhere to all general requirements

75 that will be detailed below in the Methods section.

There are a variety of different databases available to aid researchers such as RedCap [18],

77 Deduce [19], HID [20], DFBIdb [21], LONI [22], MIND [23], NeuroLOG [24], etc. We elected

78 to customize the Longitudinal Online Research and Imaging System (LORIS) [25-28] to help

79 organize data and facilitate international collaborations when conducting multi-site natural history

80 studies because of its strong track record and the fact that it is open source. Here, we detail below

81 how our group used LORIS and 21 CFR Part 11 guidelines to set up workflows and developed the

82 LORIS MyeliNeuroGene Database for Rare Diseases to lead us to clinical trial preparedness in the 83 coming years.

\section{Results}

An iteration of LORIS was installed and configured for the MyeliNeuroGene Research

87 Group at the Research Institute of the McGill University Health Centre. This database is easily

88 accessible via a web browser and multi-modal, with the ability to capture genetics data, medical

89 history, medical imaging, detailed assessments of cognition and motor function, and patient-

90 derived outcomes, among other things.

Within LORIS, data entry forms, or instruments, were created using the "Instrument

92 Builder" module. Using the workflow found in the Methods section, 90 LORIS instruments were 
94 clinical evolution, time to event, neurological examination, neuropsychological assessment, etc.

95 were developed in conjunction with other parent- and patient-reported outcomes such as quality

96 of life, disability, and stress. Of the 90 instruments, 62 had scoring algorithms developed to aid in

97 data processing. The resulting instruments are summarized in Table 1.

Table 1: Developed Instruments of the MyeliNeuroGene Loris Database

\begin{tabular}{|c|c|}
\hline Instrument & Purpose \\
\hline Family History & Inheritance pattern \\
\hline Perinatal History & Disease Onset/Progression \\
\hline Developmental History & Disease Onset/Progression \\
\hline Investigations & Diagnostic Odyssey \\
\hline Demographics & Sociodemographic variables \\
\hline Clinical Presentation & Disease Onset/Progression \\
\hline Primary Diagnosis & Disease Onset/Progression \\
\hline Gross Motor Function Measure - 88 & Measure changes in motor function \\
\hline Leiter-3 Intelligence Scale & Measure changes in intelligence \\
\hline Neuropsych Examinations & Measure changes in cognition \\
\hline Rehabilitation & PT, OT, SLT, etc. used \\
\hline Clinical Evolution & Disease Onset/Progression \\
\hline Time to Event & Disease Milestones \\
\hline Clinical Examination & Disease Onset/Progression \\
\hline Swallowing Scales & VFSS and FEES evaluation \\
\hline MRI Analyses & Disease Onset/Progression \\
\hline Modified Ashworth Scale (MAS) & Measure changes in spasticity \\
\hline Fahn Marsden Scale (F-M) & Measure changes in dystonia \\
\hline Global Dystonia Scale (GDS) & Measure Changes in dystonia \\
\hline Guy's Neurological Disability Scale (GNDS) & Measure disability and ADL \\
\hline Gross Motor Function Classification System (GMFCS) & Characterize gross motor function \\
\hline Communication Function Classification System (CFCS) & Characterize communication function \\
\hline Manual Ability Classification System (MACS) & Characterize fine motor function \\
\hline Eating and Drinking Ability Classification System (EDACS) & Characterize eating function \\
\hline Scale for the Assessment and Rating of Ataxia & Measure changes in ataxia \\
\hline Non-communicating Children's Pain Checklist - Revised & Measure parent reported pain \\
\hline Parent Reported Stress Questionnaires & Measure parental stress \\
\hline Health-Related Quality of Life Questionnaires & Measure patient's quality of life \\
\hline
\end{tabular}


99 Abbreviations: PT: physical therapy; OT: occupational therapy; SLT: Speech and language therapy; 100 VFSS: Video fluoroscopic swallow study; FEES: Fiberoptic endoscopic evaluation of swallowing; MRI: 101 Magnetic resonance imaging; ADL: Activities of daily living

102 One thousand patients and family members with rare diseases have been included into 103 LORIS and assigned unique identifiers. This includes activation of enrollment, informed consent 104 designation, external identifier logging, and family relationship mapping.

In addition, a dynamic letter generator is currently in development to assist in forwarding 106 patient information to other physicians. The tool compiles the patient's data, entered via the 107 phenotyping instruments, into a Clinical Examination Letter. In place of the database field names, 108 highlighted in yellow in Figure 1, an instance of the letter renders the patient data for the 109 corresponding field. The Clinical Examination Letter can be exported as an editable word 110 document that details patient information, such as family history, clinical evolution, time to event 111 and future plans for investigations. This letter can then be sent to the referring physicians for 112 continuity of care, and has the advantage of not duplicating work done by the data entry clinician; 113 as the clinician sees the patient and enters the data in the LORIS MyeliNeuroGene Database, the 114 clinical note is auto-populated. 
DATE OF THE VISIT: \{\$DateFromLastVisit\}

REFERRINGMD: Dr. \{\$ClinicalPresentation/Q02Presentation\}

CC: $\{$ ClinicalPresentation $/$ Q02Presentation $\}$

RE: $\{$ NameOfPatient

$\mathrm{MCH} \#$ : $\{$ MCHNUMBER

D.O.B.: $\{\$ \mathrm{DOB}\}$

\section{CONSULTATION FROM THE LEUKODYSTROPHIES AND} NEUROMETABOLIC DISORDERS CLINIC

Dear Dr. \{\$ClinicalPresentation/Q02Presentation\},

Thank you for refering this $\{\$ D O B\}$ year/month-old \$\$ClinicalPresentation/Q04Presentation, Q03 Presentation\}-handed to the Leukodystrophies and Neurometabolic Disorders clinic. The patient was seen on $\{\$ D$ ateFromLastVisit\}. The patient came with OneOrBoth parents.

Youreferred the patient for $\{\$ C$ linicalPresentation/Q01 Presentation\}.

Family History: $\{$ NameOfPatient is the $\{\$$ FamilyHistory/Q02FamilyHistory $\}$ of \{\$FamilyHistory/Q03Family History\} children. They have \{\$Family History/Q05Family History\} sisters and $\{\$$ FamilyHistory/Q04FamilyHistory $\}$ brothers.

The mother is \{\$FamilyHistory/Q01MotherHistory\} years old and is \{\$FamilyHistory/Q02MotherHistory\} healthy. \{\$FamilyHistory/Q03MotherHistory\}. She has \{\$FamilyHistory/Q04MotherHistory\} had miscamiages. \{If YES Q04MotherHistory $=>$ Family History/Q05MotherHistory, Q06MotherHistory, Q07MotherHistory, Q08MotherHistory\}. She works as a \{\$FamilyHistory/Q09MotherHistory\}. The matemal family is from $\{\$$ Family History/Q1 0 MotherHistory\}. The mother's last name is $\{$ MotherLastName. The matemal grandmother is from $\{\$$ Family History/Q03ExtendedFamily $\}$ and is \{\$Family History/Q01 HistoryDisease\} healthy. The matemal grandfather is from \{\$FamilyHistory/Q04ExtendedFamily\} and is \{\$FamilyHistory/Q02HistoryDisease\} healthy. The patient has \{\$FamilyHistory/Q1 1MotherHistory\} matemal aunts and \{\$FamilyHistory/Q12MotherHistory\} matemal uncles and has \{\$Family History/Q13MotherHistory\} cousins on their mother's side of the family.

Figure 1: Screenshot of the LORIS MyeliNeuroGene dynamic letter generator: Yellow highlights customizable variables for the clinical letter generator. Black highlighted variables represent information that is not stored in LORIS and must be filled in by the physician.

\section{Discussion}

Most patients affected with rare diseases, from mildly to severely affected, support data sharing to promote research, healthcare, and knowledge transfer [17]. We have built and customized a LORIS database and detailed our workflow to aid rare disease researchers to create their own information management system, electronic health records, or database. There is a major need and benefit to sharing data in rare disease research. De-identifying and sharing information 
126 allows rare disease researchers to efficiently study disorders by collaborating and minimizing 127 redundant studies [29], and by maximizing sample sizes.

An exportable dynamic letter generator has also been developed to save time when examining patients referred to the clinic. Patients with a rare disease who come to the Montreal Children's Hospital undergo a battery of tests that can take up to two days to complete. All information is stored in the LORIS MyeliNeuroGene Database and can be exported in the form of a Clinical Examination Letter detailing all results, impressions, and plans to help treat the patients. This letter is then sent back to the referring physician for continuity of care. When this letter is written by hand it takes a few hours and introduces numerous chances for human error. Exporting the letter from quality-controlled instruments reduces this error and saves researchers and physicians' time.

In addition to the clinical phenotyping instruments and dynamic letter generator, we have outlined, for the first time, the methodology to become Title 21 Code of Federal Regulations Part 11 Compliant, which is a requirement to use electronic records as historical controls in clinical trials in the United States $[30,31]$. To our knowledge, our manuscript is the first to outline the requirements to adhere to 21 Code of Federal Regulations Part 11 Compliance. Future work will leverage the tools developed in this project to delineate the natural history of several rare diseases and will hopefully be used by clinicians and researchers around the globe.

\section{Conclusions}

A major obstacle in rare disease research is overcoming small cohorts. Developing an online database that international collaborators can access and contribute to from all over the world is invaluable for increasing cohort sizes, discerning surrogate markers, and improving natural 
149 history data. Using this FDA compliant natural history data to validate outcome measures will be

150 life-changing for patients and families because it will lead to historical control data that can be 151 used in emerging clinical trials.

\section{Methods}

\section{Title 21 Code of Federal Regulations Part 11 Compliance (Part 11 Compliance)[32]} been customized to include additional security measures such as time stamped audit trails. We are

157 currently implementing the electronic signatures and the 2-factor authentication. There is a gap in 158 scientific literature detailing workflow and database development. As such, we will summarize the 159 general requirements of Part 11 Compliance below and how they were implemented into our 160 database.

Training Verification: Users are required to have their credentials (e.g. education, training,

162 experience) verified before performing tasks within the database. Written policy must be signed 163 holding users accountable and responsible for their electronic signatures (discussed further below).

164 This written policy must be stored, and a hard copy sent to the Office of Regional Operations 165 (HFC-100), 5600 Fishers Lane, Rockville, MD 20857.

Biometrics: This is a method of verifying an individual's identity based on a measurement

167 of the individual's physical features (i.e. fingerprints, etc.) or repeatable action that are unique to 168 that person. In our case, we chose to use a unique pin separate from an authorized user's password 169 for 2-factor authentication. 
171 access to the system is controlled by the same people who are responsible for the content of the

172 electronic records. This includes the researchers and principal investigator. Operational audits on

173 the system are done on a routine basis. Time stamp audit trails are tracked for each authorized user

174 to trace creation, modification, or deletion of any instrument, visit, or other electronic record. User

175 access is hierarchical, meaning some users do not have full access to the database and may only

176 have "read" or "write" access only. The database also must ensure that no user has the same pin

177 or password, and that pins and passwords are periodically checked and changed to prevent 178 unauthorized use. If unauthorized use occurs, there are immediate system security notifications. 179 Per Canadian predicate rules, records must be stored for 25 years after study completion. United 180 States record retention rules require storage for a minimum of 10 years.

Quality Control: Processing pipelines must ensure data fits specific parameters and types. This is discussed in depth under the Methods section "LORIS Database and Workflow".

Electronic signature: This includes any combination of text, graphic, data, audio, or other information that is represented in digital form by the database. Electronic signatures must include printed names of the signers, dates and times, meanings (e.g. approval, creation, reviewing), and an internal audit trail. These signatures are legally binding. Authority checks are completed every month to ensure only authorized users may sign, input, output, or modify records.

Digital signature: A digital signature combines the electronic signature and its corresponding cryptographic authentication, usually a pin and/or password that is used to verify 190 the identity of the signer. It cannot be copied or pasted to or from another document, making it 191 inexorably linked to the signed document. To not become cumbersome, continuous signing periods 192 only require the first to be 2 factors authenticated with a biometric identification and password. 
External Auditing: It is highly recommended that after database development a third-party

194 auditor inspects the system and documentation put in place. Auditors alert parties of any gaps or

195 shortcomings and can advise developers of what needs to be changed for full compliance with

196 local and international regulations. This will be organized for the MyeliNeuroGene database.

\section{LORIS Database and Workflow}

Architecture: LORIS is a web-based data and project management software that stores

199 demographic, clinical, behavioral, genetic, imaging, and patient-related outcomes accessible from

200 any computer browser connected to the internet [25]. Multiple sites can enter, organize, and

201 validate data under one management framework. Longitudinal data is organized around the 202 "Subject Profile". Clinical examination, imaging data, outcome measures, and metadata are 203 organized by "Visits". All stored information is de-identified and can be queried by an authorized 204 user. Source documentation can be uploaded and affiliated with each visit. Quality control is 205 ensured by automated scoring of clinical, behavioral and patient-reported outcomes, validating 206 data types (string vs numerical), and requiring double data entry where necessary.

208 dictionary in the form of an Excel sheet. This spreadsheet outlined all of the data entry forms, or 209 instruments, that would be developed using the LORIS Instrument Builder module detailed below. 210 After instrument creation, participant enrollment and data entry can begin, with query and 211 dissemination details tackled later. An overview of the workflow can be found in Figure 2. 


\section{Instrument Creation}

- Draft demographic, clinical, behavioral, genetic, imaging, and patient-related outcome data entry forms in Excel

- Use "Instrument Builder" module in LORIS to create the instrument files

- Develop PHP scoring scripts for automated scoring

\section{Participant Enrollment and Data Entry}

- Retrospectively enroll patient and family members with different rare diseases recruited previously

- Participants have profiles activated, informed consent forms logged, external identifiers noted, and family relationships mapped

- Prospectively enroll patients and family members

\section{Clinical Examination Letter}

- Use phenotyping instruments and variables, export editable text document for physicians' notes.

- Information included: family history, neurological examination, imaging and genetic investigations, medication use, and future plans for patients.

\section{Figure 2: Database development workflow to create instruments, scoring algorithms, enroll} patients, enter data, and output information into a clinical examination letter

Instrument Builder: Within LORIS are different modules to help researchers with no

217 of demographic, clinical phenotyping, behavioral, genetic, imaging, and patient-related outcome

218 measures. Each instrument can be customized with specific information such as a "Header",

219 "Label", and "Scored Field" that give the instrument title, background information, and

220 automatically calculated scoring respectively.

Data entry can be standardized using a "Textbox", "Text area", "Dropdown",

222 "Multiselect", "Date", and "Numeric" question entry. Each question is assigned a variable name

223 "Question Name", for calculations and data querying, and "Question Text" which asks the 
224 pertinent question at hand. For Dropdown questions, instrument specific options can be added for 225 every question.

226 Instrument Creation: Instruments were first planned and drafted using Excel in the form of

227 a Data Dictionary. Columns consisted of Question Names, type of question (e.g. Numeric, 228 Dropdown, etc.), Question Text, Question Options (available choices), and Formulas (for later 229 calculations). Each row represented one question. Using the Data Dictionary and the Instrument 230 Builder module on LORIS, each instrument was created: demographic forms, clinical phenotyping 231 (i.e. spasticity and dystonia measures, gross and fine motor, eating, and drinking function, ataxia, 232 intelligence, disability, swallowing evaluations etc.), behavioral, genetic, imaging (i.e. MRI 233 analyses), and patient-related outcomes (i.e. health-related quality of life, parental stress, pain 234 characterization, etc.). Instruments' files were then uploaded onto the MyeliNeuroGene private 235 repository on GitHub as Pull Requests for review.

236 Scoring Algorithms: After instrument completion, a PHP scoring script was developed for 237 instruments that required them. Automatic scoring reduces human error and dramatically decreases 238 time spent on calculations. Scoring scripts were also uploaded onto the GitHub repository for 239 review.

Instrument Implementation: After instruments and scoring scripts were reviewed and 241 modified, the Pull Requests on GitHub were approved and the instruments made available on an 242 insulated LORIS staging server where beta testing occurred. After testing was completed, 243 instruments were pushed to the LORIS production server for instrument pipeline completion and 244 data entry. 
Participant Enrollment: Before data entry could be completed, Subject Profiles had to be

entered. Our group has consented more than 1,000 patients and family members with different rare diseases since 2011, and patient and family recruitment is ongoing. To create a new profile, "Date of Birth", "Sex", "Site" (in the case of a multi-site study), and "Project" must be entered. Projects can be separated into different studies such as natural history, imaging, genetic, or even clinical trials assessing therapeutics. A new Subject Profile, or candidate, generates two identifier codes, a DCCID and a PSCID which are unique LORIS identifiers.

After the creation of the Subject Profile, each candidate was activated in the study, designated for which informed consent form was signed, and mapped to any external identifier codes. Under "Participant Status", we tracked the participant's status in the study (e.g. Active, Death, Lost to Follow-up, etc.). Comments can be entered with both time, date, and author history tracked in the internal audit trail. "Consent Status" tracks the latest signed Research Ethics Board (REB) approved informed consent form. Finally, mapping the "External Identifier" is crucial for future correspondence with family doctors and other collaborators.

Data Entry: "Create time point" allows for data entry of clinical, behavioral, and patient determined outcomes that were created during the Instrument Creation process. It also enables uploading of any imaging data collected. We customized our time points to correspond to the age of the patient. For instance, a participant's birth date would be time point T000, and a follow-up appointment 6 months later would be time point T006. A prenatal examination 1 month before a T000 examination would be designated as T-001. The steps to creating a time point can be seen in Figures 3, 4, and 5. 


\begin{tabular}{|c|c|c|c|c|c|c|c|c|c|c|c|}
\hline \multicolumn{4}{|l|}{ Dов } & \multicolumn{4}{|c|}{ Biological Sex } & \multicolumn{4}{|l|}{ Project } \\
\hline \multicolumn{4}{|l|}{ 1800-01-01 } & \multicolumn{4}{|l|}{ Male } & \multicolumn{4}{|l|}{ Myelineurogene } \\
\hline \multicolumn{12}{|l|}{ Actions: } \\
\hline Create time point & Edit Candidate Info & Family & formation & \multicolumn{3}{|c|}{ View Imaging datasets } & & & & & \\
\hline \multicolumn{12}{|c|}{ List of Visits (Time Points) } \\
\hline $\begin{array}{l}\text { Visit Label } \\
\text { (Click to Open) }\end{array}$ & Subproject & Site & Stage & Stage Status & Date of Stage & Sent To DCC & Imaging Scan Done & Feedback & BVL QC & BVL Exclusion & Registered By \\
\hline
\end{tabular}

\section{Figure 3: Creating longitudinal time points for patient visits}

\section{n $\rangle$ Access Profile $\rangle$ Candidate Profile $343247 /$ MTL0007 $\rangle$ Create Time Point \\ Create Time Point}

DCCID

Subproject

Site

Visit label

343247

Leukodystrophy and Leukoencephalo $\mathbf{v}$

Center Universitaire de Santé McGill v

T000

$\mathbf{v}$

\section{Create Time Point}

Figure 4: Associating time points with subprojects and study sites

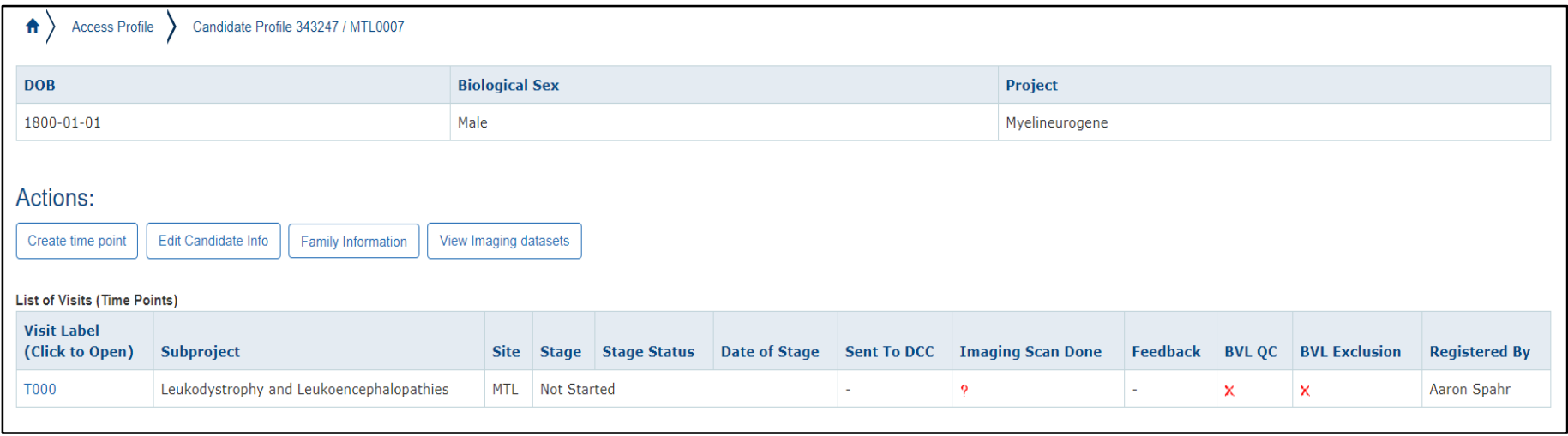


Figure 6: Test battery of instruments customized for each participant based on time point and age appropriateness

Family Information: We have further customized LORIS to include Family Relationship 279 information. Linking de-identified individuals allows us to link a given patient's disease 280 characteristics to his/her parents' reported measures such as parental stress or 281 patient/parents/sibling's quality of life. It also allows us to organize family genetic results when 282 next generation sequencing (NGS) investigations are being conducted as well as any family/parent 283 reported outcomes.

\section{Declarations}

Ethics approval and consent to participate: Written and informed consent was obtained 286 from all research participants. This study was approved by the Research Ethics Board of the McGill 287 University Health Center Research Institute (11-105-PED, 2019-4972) 
Availability of data and materials: The datasets used and/or analyzed during the current

290

study are available from the corresponding author on reasonable request.

Competing interests: Our group, the MyeliNeuroGene Lab, is a collaborator of Dr. Alan C Evans' research group, the McGill Centre for Integrative Neuroscience, who developed LORIS, a free and open-source web-accessible database solution for multi-modal data and multi-site studies.

Funding: The authors disclosed receipt of the following financial support for the research, authorship, and/or publication of this article : This work was supported by the Montreal Children's Foundation (Estate of Daphne Dale Townsend), Fondation Les Amis d'Elliot, Fondation le Tout pour Loo, and Leuco-Action. Dr. Bernard has received the New Investigator Salary Award from the Canadian Institutes of Health Research (2017-2022). Aaron Spahr has received funding from the Desjardins Studentship in Child Health Research through the Research Institute of McGill University Health Centre (2018-2019), the Healthy Brains for Healthy Lives Graduate Student Fellowship (2019-2020), as well as a Graduate Excellence Award from the Integrated Program in Neuroscience at McGill University (2018-2019). None of the funding sources was relevant for study design, collection of data, analysis and interpretation of data, or writing of this manuscript.

Author's contributions: AS, ZR, ML, SD, ACE, GB were responsible for the conception and design of this project. AS developed, all of the instruments, with contributions from HT and expert guidance from GB, ZR, LD and ML. AS, ZR, and ML developed the scoring scripts. CL and MS helped perform quality control on the scoring scripts. CM developed the imaging platform. AS retrospectively entered over 1,000 participants and was responsible for initial data entry. AS generated the figures and the tables, with contributions from SF. AS drafted the initial manuscript, with contributions and modifications from SF and LT. ZR, ML, CM, SD, LT, SF, HT, LD, ACE, and GB provided feedback and all authors approved the current submitted version. 
313 participation, time, and patience to complete questionnaires. The authors also wish to thank all

314 collaborators and clinicians who referred patients, research would not be possible without you.

315 Author's information: GB is a pediatric neurologist and clinician scientist leading the

316 Leukodystrophy and Neurometabolic Disorders Clinic at the McGill University Health Centre

317 Research Institute.

318 List of Abbreviations
ADL Activities of Daily Living
CFCS Communication Function Classification System
CFR Code of Federal Regulations
DCCID LORIS Identifier
Eating and Drinking Ability Classification
EDACS System
FDA Food and Drug Administration
FEES Fiberoptic Endoscopic Evaluation of Swallowing
GDS Global Dystonia Scale
GMFCS Gross Motor Function Classification System
GNDS Guy's Neurological Disability Scale
HID Human Clinical Imaging Database
LORIS Longitudinal Online Research and Imaging System
LONI LONI Image Data Archive
MACS Manual Ability Classification System
MAS Modified Ashworth Scale
MRI Magnetic Resonance Imaging
NGS Next Generation Sequencing
OT Occupational Therapist
PHP PHP: Hypertext Preprocessor
PSCID LORIS Identifier
PT Physical Therpist
REB Research Ethics Board
SaLT Speech and Language Therapist
VFSS Video Fluoroscopic Swallow Study 
322 1. Aymé S, Urbero B, Oziel D, Lecouturier E, Biscarat AC. Information on rare diseases: the Orphanet 323 project. La Revue de médecine interne. 1998;19:376S-7S.

324 2. Baird PA, Anderson TW, Newcombe HB, Lowry RB. Genetic disorders in children and young 325 adults: a population study. American journal of human genetics. 1988;42(5):677.

326 3. Humphreys G. Coming together to combat rare diseases. SciELO Public Health; 2012.

327 4. McKusick VA. Mendelian Inheritance in Man and its online version, OMIM. The American 328 Journal of Human Genetics. 2007;80(4):588-604.

329 5. Wakap SN, Lambert DM, Olry A, Rodwell C, Gueydan C, Lanneau V, et al. Estimating cumulative 330 point prevalence of rare diseases: analysis of the Orphanet database. European Journal of Human Genetics. $331 \quad 2019: 1-9$.

332 6. Boycott KM, Vanstone MR, Bulman DE, MacKenzie AE. Rare-disease genetics in the era of next333 generation sequencing: discovery to translation. Nature Reviews Genetics. 2013;14(10):681.

334 7. Sawyer SL, Hartley T, Dyment DA, Beaulieu CL, Schwartzentruber J, Smith A, et al. Utility of 335 whole-exome sequencing for those near the end of the diagnostic odyssey: time to address gaps in care. 336 Clinical genetics. 2016;89(3):275-84.

337 8. E Samuels M. Saturation of the human phenome. Current genomics. 2010;11(7):482-99.

338 9. Sardana D, Zhu C, Zhang M, Gudivada RC, Yang L, Jegga AG. Drug repositioning for orphan 339 diseases. Briefings in bioinformatics. 2011;12(4):346-56.

340 10. Griggs RC, Batshaw M, Dunkle M, Gopal-Srivastava R, Kaye E, Krischer J, et al. Clinical research 341 for rare disease: opportunities, challenges, and solutions. Molecular genetics and metabolism. $342 \quad 2009 ; 96(1): 20-6$.

343 11. Helman G, Van Haren K, Bonkowsky JL, Bernard G, Pizzino A, Braverman N, et al. Disease 344 specific therapies in leukodystrophies and leukoencephalopathies. Molecular genetics and metabolism. $345 \quad 2015 ; 114(4): 527-36$. 
347 Introduction to Applied Epidemiology and Biostatistics. cdc.gov: U.S. Department of Health and Human 348 Services; 2006.

349 13. Hobbs BP, Sargent DJ, Carlin BP. Commensurate priors for incorporating historical information in 350 clinical trials using general and generalized linear models. Bayesian analysis (Online). 2012;7(3):639.

351 14. Neuenschwander B, Capkun-Niggli G, Branson M, Spiegelhalter DJ. Summarizing historical 352 information on controls in clinical trials. Clinical Trials. 2010;7(1):5-18.

353 15. Pocock SJ. The combination of randomized and historical controls in clinical trials. Journal of 354 chronic diseases. 1976;29(3):175-88.

355 16. Viele K, Berry S, Neuenschwander B, Amzal B, Chen F, Enas N, et al. Use of historical control 356 data for assessing treatment effects in clinical trials. Pharmaceutical statistics. 2014;13(1):41-54.

357 17. Courbier S, Dimond R, Bros-Facer V. Share and protect our health data: an evidence based 358 approach to rare disease patients' perspectives on data sharing and data protection - quantitative survey and 359 recommendations. Orphanet journal of rare diseases. 2019;14(1):175.

360 18. Harris PA, Taylor R, Thielke R, Payne J, Gonzalez N, Conde JG. Research electronic data capture 361 (REDCap) — a metadata-driven methodology and workflow process for providing translational research 362 informatics support. Journal of biomedical informatics. 2009;42(2):377-81.

363 19. Horvath MM, Winfield S, Evans S, Slopek S, Shang H, Ferranti J. The DEDUCE Guided Query 364 tool: providing simplified access to clinical data for research and quality improvement. Journal of 365 biomedical informatics. 2011;44(2):266-76.

366 20. Ozyurt IB, Keator DB, Wei D, Fennema-Notestine C, Pease KR, Bockholt J, et al. Federated web367 accessible clinical data management within an extensible neuroimaging database. Neuroinformatics. $368 \quad 2010 ; 8(4): 231-49$.

369 21. Adamson CL, Wood AG. DFBIdb: a software package for neuroimaging data management. 370 Neuroinformatics. 2010;8(4):273-84. 
371 22. Dinov I, Van Horn J, Lozev K, Magsipoc R, Petrosyan P, Liu Z, et al. Efficient, distributed and

372 interactive neuroimaging data analysis using the LONI pipeline. Frontiers in neuroinformatics. 2009;3:22.

373 23. Bockholt HJ, Scully M, Courtney W, Rachakonda S, Scott A, Caprihan A, et al. Mining the mind

374 research network: a novel framework for exploring large scale, heterogeneous translational neuroscience

375 research data sources. Frontiers in neuroinformatics. 2010;3:36.

376 24. Gibaud B, Kassel G, Dojat M, Batrancourt B, Michel F, Gaignard A, et al., editors. NeuroLOG:

377 sharing neuroimaging data using an ontology-based federated approach. AMIA Annual Symposium

378 Proceedings; 2011: American Medical Informatics Association.

379 25. Das S, Zijdenbos AP, Harlap J, Vins D, Evans AC. LORIS: a web-based data management system 380 for multi-center studies. Frontiers in neuroinformatics. 2011;5:37.

381 26. Das S, Glatard T, MacIntyre LC, Madjar C, Rogers C, Rousseau ME, et al. The MNI data-sharing 382 and processing ecosystem. Neuroimage. 2016;124(Pt B):1188-95.

383 27. Das S, Glatard T, Rogers C, Saigle J, Paiva S, MacIntyre L, et al. Cyberinfrastructure for Open 384 Science at the Montreal Neurological Institute. Frontiers in Neuroinformatics. 2017;10(53).

385 28. Das S, Lecours Boucher X, Rogers C, Makowski C, Chouinard-Decorte F, Oros Klein K, et al. 386 Integration of “omics” Data and Phenotypic Data Within a Unified Extensible Multimodal Framework. 387 Frontiers in Neuroinformatics. 2018;12(91).

388 29. El Emam K, Rodgers S, Malin B. Anonymising and sharing individual patient data. bmj. $389 \quad 2015 ; 350: \mathrm{h} 1139$.

390 30. Administration USFaD. Guidance for Industry: Part 11, Electronic Records; Electronic Signatures 391 - Scope and Application. In: Services USDoHaH, editor. fda.gov: U.S. Department of Health and Human 392 Services; 2003. p. 12.

393 31. Administration UFaD. Rare diseases: natural history studies for drug development guidance for 394 industry fda.gov: U.S. Department of Health and Human Services; 2019 [Available from: 395 https://www.fda.gov/media/122425/download. 
396 32. Administration USFD. Electronic Code of Federal Regulations [Webpage]. 62 FR 13464: U.S.

397 Department of Health \& Human services; 1997 [updated January 14, 2020.

398 


\section{Figures}

DATE OF THE VISIT: \{\$DateFromLastVisit\}

REFERRINGMD: Dr. \{SClinicalPresentation Q02Presentation\}

$\mathrm{CC}:\{$ SClinicalPresentation Q02Presentation\}

RE: (NameOfPatient

$\mathrm{MCH} \#$ : $\{\mathrm{MCHNUMBER}$

D.O.B.: $\{$ SDOB $\}$

\section{CONSULTATION FROM THE LEUKODYSTROPHIES AND \\ NEUROMIETABOLIC DISORDERS CLINIC}

Dear Dr. \{\$ClinicalPresentation Q02Presentation\},

Thank you for referning this \{SDOB\} year/month-old \{SClinicalPresentation Q04Presentation, Q03Presentation\}-handed, to the Leukodystrophies and Neurometabolic Disorders clinic. The patient was seen on \{SDateFromLastVisit\}. The patient came with OneOrBoth parents.

Youreferred the patient for $\{\$$ ClinicalPresentation/Q01 Presertation\}.

Family History: \{NameOfPatient is the \{SFamilyHistory Q02FamilyHistory\} of \{\$FamilyHistory Q03Family History\} children. They have \{SFamily History Q05Family History\} sisters and \{\$Family History/Q04Family History $\}$ brothers.

The mother is \{\$Family History/Q01Mother History\} years old and is \{\$Family History/Q02MotherHistory\} healthy. \{SFamily Fistory/Q03MotherHistory\}. She has \{SFamily History/Q04MotherHistory\} had miscamiages. \{If YES Q04MotherHistory $\Rightarrow$ Family History/Q05MotherHistory, Q06MotherHistory, Q07MotherHistory, Q08MotherHistory\}. She works as a \{SFamily History/Q09MotherHistory\}. The matemal family is from \{\$Family History/Q10MotherHistory\}. The mother's last name is MotherLastName. The matemal grandmother is from \{SFamily History Q03ExtendedFamily\} and is \{\$Family History/Q01History Disease\} healthy. The matemal grandfather is from \{SFamilyHistory/Q04ExtendedFamily\} and is \{SFamilyHistory/Q02 HistoryDisease\} healthy. The patient has \{SFamily History/Q1 1MotherHistory\} matemal aunts and \{SFamilyHistory/Q1 2MotherHistory\} matemal uncles and has \{SFamily History Q13MotherHistory\} cousins on their mother's side of the family.

\section{Figure 1}

creenshot of the LORIS MyeliNeuroGene dynamic letter generator: Yellow highlights customizable variables for the clinical letter generator. Black highlighted variables represent information that is not stored in LORIS and must be filled in by the physician. 


\section{Instrument Creation}

- Draft demographic, clinical, behavioral, genetic, imaging, and patient-related outcome data entry forms in Excel

- Use "Instrument Builder" module in LORIS to create the instrument files

- Develop PHP scoring scripts for automated scoring

\section{Participant Enrollment and Data Entry}

- Retrospectively enroll patient and family members with different rare diseases recruited previously

- Participants have profiles activated, informed consent forms logged, external identifiers noted, and family relationships mapped

- Prospectively enroll patients and family members

\section{Clinical Examination Letter}

- Use phenotyping instruments and variables, export editable text document for physicians' notes.

- Information included: family history, neurological examination, imaging and genetic investigations, medication use, and future plans for patients.

\section{Figure 2}

Database development workflow to create instruments, scoring algorithms, enroll patients, enter data, and output information into a clinical examination letter

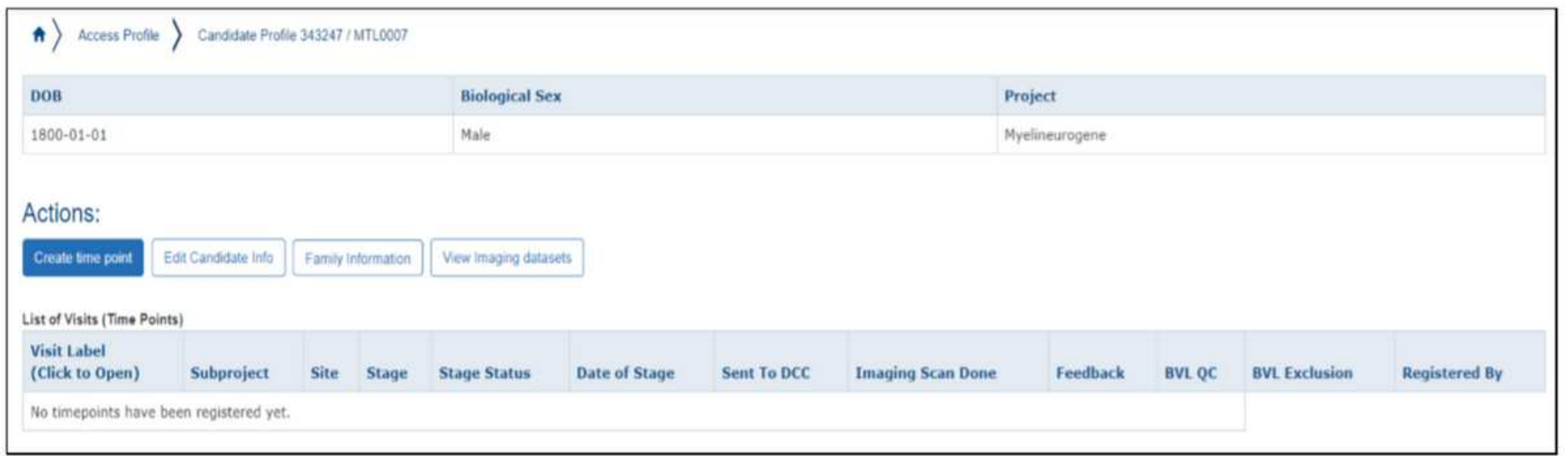

Figure 3

Creating longitudinal time points for patient visits 


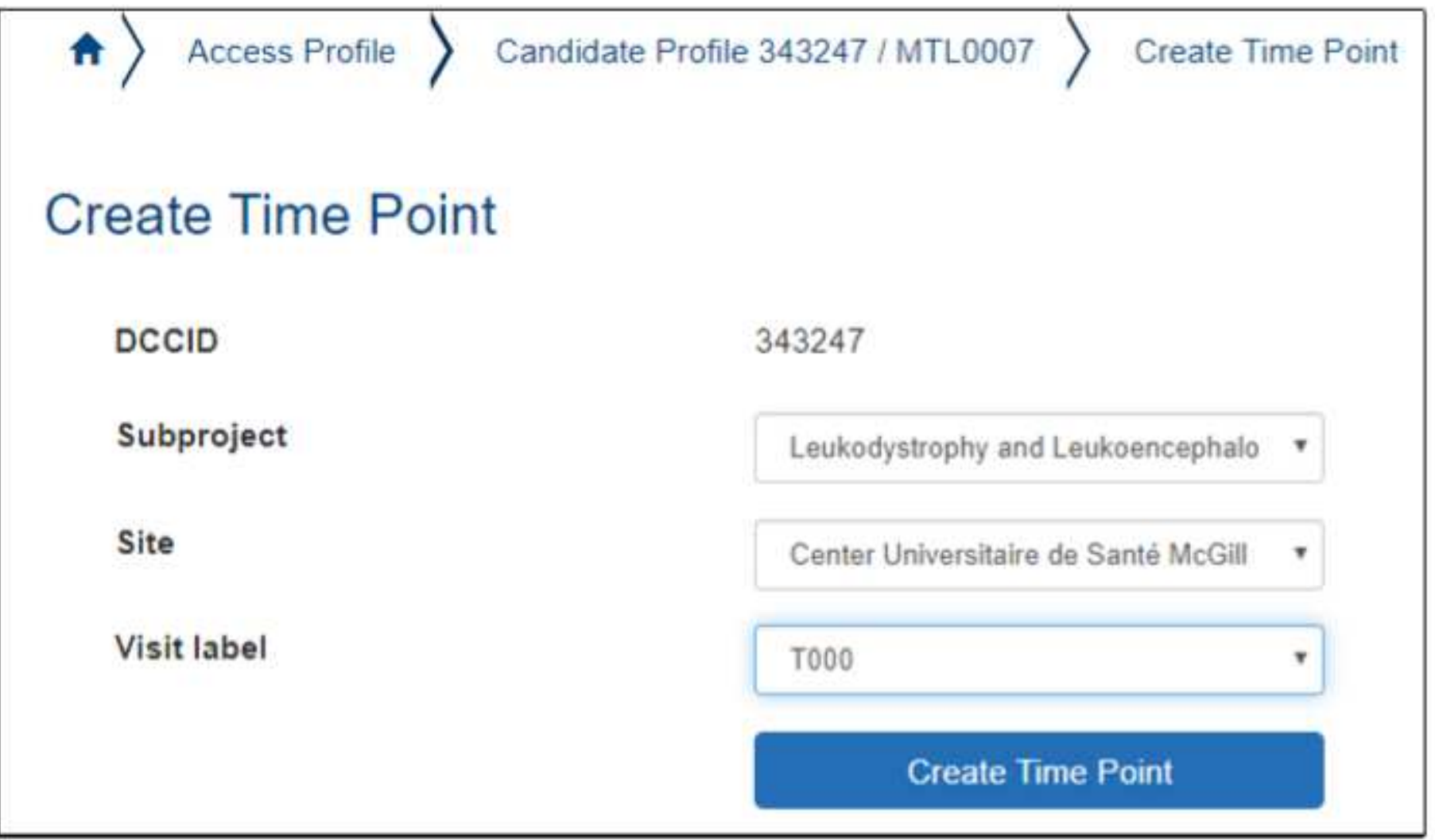

\section{Figure 4}

Associating time points with subprojects and study sites

\begin{tabular}{|c|c|c|c|c|c|c|c|c|c|c|c|c|c|c|}
\hline Dов & & & & logical & Sex & & & & & Project & & & & \\
\hline 1800-01-01 & & & & & & & & & & Myelineurogene & & & & \\
\hline \multicolumn{15}{|l|}{ Actions: } \\
\hline Create line point & Eoril Candidate into & Fanily Information & \multicolumn{3}{|c|}{ View inuging datasets } & & & & & & & & & \\
\hline \multicolumn{15}{|c|}{ List of Visits (Time Points) } \\
\hline $\begin{array}{l}\text { Visit Label } \\
\text { (Click to Open) }\end{array}$ & \multicolumn{3}{|l|}{ Subproject } & Site & Stage & Stage Status & Date of Stage & Sent To DCC & \multicolumn{2}{|c|}{ Imaging Scan Done } & Feedback & BVL QC & BVL Exclusion & Registered By \\
\hline To00 & \multicolumn{3}{|c|}{ Leukodystrophy and Leukoencephalopathies } & MTL & \multicolumn{3}{|c|}{ Not Started } & & ? & & & $\mathrm{x}$ & $\mathrm{x}$ & Aaron Spahr \\
\hline
\end{tabular}

\section{Figure 5}

Visualizing time point information in the LORIS Candidate Profile 


\begin{tabular}{|c|c|c|c|c|c|}
\hline \multicolumn{6}{|c|}{ Behavioral Battery of Instruments } \\
\hline $\begin{array}{l}\text { Instrument } \\
\text { (Click To Open) }\end{array}$ & Data Entry & Administration & Feedback & Double Data Entry form & Double Data Entry Status \\
\hline Family History & In Progress & & & & \\
\hline Perinatal History & & & - & & \\
\hline Developmental History & & & - & & \\
\hline Investigations & & & - & & \\
\hline Molecular Genetics & & & & & \\
\hline Demographics & & & & & \\
\hline
\end{tabular}

\section{Figure 6}

Test battery of instruments customized for each participant based on time point and age appropriateness 